\title{
Adaptação escolar para crianças com Transtorno do Espectro Autista (TEA)
}

\author{
School adaptation for children with Autism Spectrum Disorder (ASD) \\ Adaptación escolar para niños con Trastorno del Espectro Autismo (TEA)
}

Walleska Suellen do Nascimento Silva

ORCID: https://orcid.org/0000-0002-6696-3569

Centro Universitário Unifacid Wyden, Brasil

E-mail: walleskasuellen@gmail.com

Alice Lima Rosa Mendes

ORCID: https://orcid.org/0000-0002-1960-9647

Universidade de Brasília, Brasil

E-mail: alice_lima@@hotmail.com

Rodrigo Feitosa de Oliveira Correia

ORCID: https://orcid.org/0000-0002-8614-5555

Centro Universitário Unifacid Wyden, Brasil

E-mail: rodrigofeitosatsb@gamail.com

Samyres Batista De Medeiros

ORCID: https://orcid.org/0000-0001-5647-5783

Centro Universitário Uninovafapi, Brasil

E-mail samyresmed28@gmail.com

Gabriela Dantas Carvalho

ORCID: https://orcid.org/0000-0002-9571-3323

Universidade Federal do Delta do Parnaíba, Brasil

E-mail: ftgabrieladantas@hotmail.com

Ivani Feitosa de Oliveira

ORCID: https://orcid.org/0000-0002-0662-8785

Universidade Federal do Piauí, Brasil

E-mail: ivannioliveira@hotmail.com

Luís Paulo Alves Ramos

ORCID: https://orcid.org/0000-0002-0196-8860

Universidade Federal de São Carlos, Brasil

E-mail: to.luispauloramos@gmail.com

Silvana Maria Véras Neves

ORCID: https://orcid.org/0000-0003-4784-9135

Universidade de Fortaleza, Brasil

E-mail: silvanafisio7@yahoo.com.br

Cristina Cardoso da Silva

ORCID: https://orcid.org/0000-0002-9490-4099

Universidade de Fortaleza, Brasil

E-mail: cristinascm31@gmail.com

Juçara Gonçalves De Castro

ORCID https://orcid.org/0000-0002-6634-3423

Universidade Estadual da Paraíba, Brasil

E-mail: castrojucara78@gmail.com

Suyane Santana Cavalcante

ORCID: https://orcid.org/0000-0002-1675-8380

Centro Universitário Unifacid Wyden, Brasil

E-mail: cavalcantesuy99@gmail.com

Bruna Correa Nolêto

ORCID: https://orcid.org/0000-0001-7371-1936

Centro Universitário Unifacid Wyden, Brasil

E-mail: brunacnoleto@gmail.com

Maria Aparecida de Souza

ORCID: https://orcid.org/0000-0003-3298-7658 Universidade Estadual de Ciências da Saúde de Alagoas, Brasil E-mail: souzzamaria@outlook.com.br

Thalyta Kelly da Silva Fontineles

ORCID: https://orcid.org/0000-0001-7377-4419 Centro Universitário Uninovafapi, Brasil
E-mail: tatafont@outlook.com

Ana Flávia Machado de Carvalho

ORCID: https://orcid.org/0000-0002-6691-4804

Universidade Estadual da Paraíba, Brasil

E-mail: anaflaviaparaibana@hotmail.com 


\begin{abstract}
Resumo
O estudo teve como objetivo, identificar a necessidade das adaptações escolares para crianças com TEA, visando facilitar o processo de ensino e aprendizagem. Trata-se de uma pesquisa de campo, predominantemente quantitativo, descritivo. Foi realizada em uma instituição Municipal de ensino em Teresina-PI, com 3 docentes, onde será necessário trabalhar na instituição a mais de um ano, e ter alunos autistas em suas turmas de aula. A coleta de dados foi realizada através da aplicação de um questionário elaborado pelo pesquisador de forma 'online', utilizando a plataforma Google Forms e enviado através do Gmail e do Outlook. As variáveis presentes nesse questionário foram: o reconhecimento do tema, a importância de realizar essas adaptações, e as dúvidas existentes pelos professores. Os dados foram submetidos a análise de conteúdo, posteriormente organizados em gráficos do programa Microsoft Excel 2016. No artigo é relatado a existência de dificuldade dos docentes ao repassar atividade para alunos com TEA, no qual (67\%) que corresponde a 2 docentes, de uma amostra de 3 professores, relatam existir com muita frequência, além de demonstrar que as crianças com TEA conseguem realizar as atividades sem adaptação adequada, com pouca frequência, segundo $100 \%$ da amostra, com isso observa-se também que os professores apresentam pouca $(67 \%)$ ou nenhuma (33\%) formação para adaptações curriculares para seus alunos com TEA. Além de ser possível verificar a frequência com que a escola não disponibiliza de materiais para que ocorra as adaptações para os alunos com TEA, onde se verifica que $67 \%$ da amostra (3 professores), afirmam ser com pouca frequência. Com isso, é notável a necessidade das adaptações, além da falta de preparo dos professores e a falta de apoio da escola, e a importância dessas adaptações para os alunos com TEA. Palavras-chave: Autismo; Adaptação escolar; Aprendizagem.
\end{abstract}

\begin{abstract}
The study aimed to identify the need for school adaptations for children with ASD, to facilitate the teaching and learning process. It is predominantly quantitative, descriptive field research. It was held in a municipal teaching institution in Teresina-PI, with 3 teachers, where it will be necessary to work at the institution for more than a year and have autistic students in their classes. Data collection was performed through the application of a questionnaire prepared by the researcher online, using the Google Forms platform and sent through Gmail and Outlook. The variables present in this questionnaire were: the recognition of the theme, the importance of making these adaptations, and the teachers' doubts. The data were subjected to content analysis, later organized in graphics of the Microsoft Excel 2016 program. The article reports the existence of difficulty for teachers to pass on activity to students with ASD, in which (67\%) corresponding to 2 teachers, of a sample of 3 teachers reported to exist very often, in addition to demonstrating that children with ASD are able to perform activities without adequate adaptation, infrequently, according to $100 \%$ of the sample, with this it is also observed that teachers have little ( $67 \%)$ or no $(33 \%)$ training for curricular adaptations for their students with ASD. In addition to being possible to verify the frequency with which the school does not provide materials for adaptations to occur for students with ASD, where it is verified that $67 \%$ of the sample ( 3 teachers) claim to be infrequently. Thus, the need for adaptations is notable, in addition to the lack of preparation of teachers and the lack of support from the school, and the importance of these adaptations for students with ASD.
\end{abstract}

Keywords: Autism; School adaptation; Learning.

\title{
Resumen
}

El estudio tuvo como objetivo identificar la necesidad de adaptaciones escolares para niños con TEA, con el fin de facilitar el proceso de enseñanza y aprendizaje. Es una investigación de campo predominantemente cuantitativa y descriptiva. Se llevó a cabo en una institución docente municipal de Teresina-PI, con 3 profesores, donde será necesario trabajar en la institución por más de un año, y tener estudiantes autistas en sus clases. La recolección de datos se realizó mediante la aplicación de un cuestionario elaborado por el investigador en línea, utilizando la plataforma Google Forms y enviado a través de Gmail y Outlook. Las variables presentes en este cuestionario fueron: el reconocimiento del tema, la importancia de realizar estas adaptaciones y las dudas de los docentes. Los datos fueron sometidos a análisis de contenido, posteriormente organizados en gráficos del programa Microsoft Excel 2016. El artículo reporta la existencia de dificultad para que los docentes transmitan la actividad a los estudiantes con TEA, en la que (67\%) correspondiente a 2 docentes, de un muestra de 3 docentes reportan muy a menudo, además de demostrar que los niños con TEA son capaces de realizar actividades sin una adecuada adaptación, infrecuentemente, según el 100\% de la muestra, con esto también se observa que los docentes tienen poca (67\%) o no (33\%) capacitación para adaptaciones curriculares para sus estudiantes con TEA. Además de ser posible verificar la frecuencia con la que la escuela no proporciona materiales para que se produzcan adaptaciones para los estudiantes con TEA, donde se verifica que el $67 \%$ de la muestra (3 profesores) afirma ser infrecuente. Así, se destaca la necesidad de adaptaciones, además de la falta de preparación de los profesores y la falta de apoyo de la escuela, y la importancia de estas adaptaciones para los estudiantes con TEA.

Palabras clave: Autismo; Adaptación escolar; Aprendiendo.

\section{Introdução}

De acordo com o Manual Diagnóstico e Estatístico de transtornos Mentais-DSM-IV (2014) o autismo é classificado como um transtorno invasivo do desenvolvimento (TID), que para a Classificação dos Transtornos Mentais e de Comportamento 
da CID-10 tem como características os indivíduos afetados pelo TID, anormalidades qualitativas nas interações sociais recíprocas e em padrões de comunicação e apresentam um repertório de interesses e atividades restritos, estereotipado e repetitivo.

O Terapeuta Ocupacional (T.O) obtém várias ferramentas para intervir com as crianças autistas, lhe trazer um maior benefício em suas atividades de vida diária, sua independência, autonomia e uma dessas ferramentas é o brincar que por este meio o sujeito obtém contato com as realidades internas e externas, sempre de forma lúdica e divertida, esta é a forma que a criança tem de se expressar, de estar no mundo, de falar de si, de conhecer o próximo, de proporcionar momento para que próximo lhe conheça e principalmente se desenvolver (Correa et al., 2017).

Para o autista ter um melhor desenvolvimento, precisa da inserção no ambiente escolar, para isso é necessário ocorrer a inclusão social e também a adaptação curricular (escolar), com a utilização desse mecanismo o professor poderá melhorar o processo de aprendizagem da criança com Transtorno do Espectro Autista(TEA), considerando não somente o conteúdo a ser ministrado, mas a forma e seu objetivo, conseguindo assim suprir as dificuldades das crianças autistas, ajudando a superar obstáculos, alcançar autonomia e um melhor processo de aprendizagem (Floro, 2019).

Os materiais utilizados para a adaptação curricular da criança autista, são pensados no individual de cada aluno e de seu currículo especificamente. Portanto, são focadas nas adaptações, estratégias comportamentais, utilizando-se de dicas visuais e recursos de comunicação suplementar e alternativa, proporcionando diferentes estímulos sensoriais e situações que possibilitem e favoreçam o processo de ensino-aprendizagem do mesmo (Silva 2016).

Diante do contexto o problema de pesquisa foi formulado com o propósito de identificar como a adaptação escolar pode ser um benefício no desempenho escolar da criança com Transtorno do Espectro Autista (TEA).

A aprendizagem é considerada um aspecto fundamental para o desenvolvimento do ser humano. Diferente do animal o ser humano (homem), ao longo da vida vai apropriando os saberes importantes dela, se inserindo no mundo simbólico da cultura transformando-se e modificando o mundo através da inserção do outro. Desta forma pode-se perceber, a importância da mediação escolar na educação de alunos com TEA, devidamente organizada e planejada pode ser fundamental para a aprendizagem dessas crianças, proporcionando um maior desenvolvimento (Benini et al.,2016).

Diante do exposto, criou-se a problemática do estudo, justificando a escolha do tema como meio de analisar a necessidade das adaptações escolares por parte dos docentes para crianças com Transtorno do Espectro Autista (TEA). O estudo poderá favorecer a sociedade, profissionais, comunidade científica, principalmente pais ou cuidadores de crianças com (TEA), através dos conhecimentos sobre o benefício da adaptação escolar, podendo proporcionar melhor desenvolvimento educacional da criança.

O objetivo geral deste estudo foi identificar a necessidade das adaptações escolares para crianças com TEA para facilitar o processo de ensino e aprendizagem, e os objetivos específicos foram, verificar o grau de conhecimento e dificuldade dos docentes sobre as atividades escolares para crianças com Transtorno do Espectro Autista, além de detectar a necessidade das intervenções adaptativas para as atividades escolares das mesma e por fim analisar se as adaptações interferem de maneira positiva no processo de aprendizagem.

\section{Metodologia}

O presente estudo realizou-se de acordo com os aspectos éticos seguindo a Resolução 466/2012 do Conselho Nacional de Saúde (CNS). A pesquisa iniciou-se a partir da aprovação do Comitê de Ética e Pesquisa (CEP) da Faculdade UniFacid.

Foi solicitada a assinatura do Termo de Consentimento Livre e Esclarecido (TCLE) pelos sujeitos da pesquisa, onde receberam por e-mail, ficando garantido o sigilo dos participantes e das informações por eles prestadas, assim como o direito de retirar o consentimento a qualquer tempo sem qualquer ônus além da autorização da instituição onde será realizada a pesquisa.

Trata-se de uma pesquisa de campo, predominantemente quantitativo, descritivo, onde analisou-se a necessidade das 
adaptações escolares para crianças com Transtorno do Espectro Autista (TEA).

A pesquisa realizou-se de forma 'online' com os docentes de uma Escola Municipal localizada na cidade de TeresinaPI, no período de outubro a novembro de 2020. A amostra foi composta por 3 professores(as).

Os critérios de inclusão: ser professor da instituição há pelo menos 1 ano, e ter alunos autistas em suas turmas de aula, para analisar seu conhecimento sobre as adaptações escolares para com seus alunos e a importância da mesma no processo de ensino e aprendizagem, e os critérios de exclusão foram: ser apenas professores temporários, Acompanhantes Terapêuticos (AT) ou professores do Atendimento Educacional Especializado (AEE) e que não respondam o questionário no prazo de um mês.

A coleta de dados foi realizada através da aplicação de um questionário elaborado pelo pesquisador de forma online, utilizando a plataforma Google Forms e enviado através do Gmail e do Outlook.

As variáveis presentes nesse questionário que foram investigadas são: o reconhecimento do tema, a importância de realizar essas adaptações, e as dúvidas existentes pelos professores. Para preservar e manter a identidade e o sigilo acerca dos dados coletados dos sujeitos da pesquisa, foram criados neste questionário, códigos de referência como: (Sujeito 1, sujeito 2, etc.). Analisaram-se as dúvidas, o conhecimento dos professores e a necessidade das adaptações no processo de ensino, após a aplicação do instrumento de coleta de dados.

Realizada a coleta, os dados foram transcritos e submetidos a análise de conteúdo, posteriormente organizados em gráficos do programa Microsoft Excel 2016. A pesquisa apresentou riscos mínimos, causando apenas desconforto nos docentes em expor seu nível de conhecimento relacionado ao assunto abordado, e pelo tempo gasto na aplicação dos questionários. Os participantes foram beneficiados, visto que a pesquisa teve o objetivo de aprimorar o conhecimento dos docentes e melhorar a relação aluno-professor, assim garantindo o melhor desempenho do aluno e uma melhor conduta dos professores em relação a encontrar alternativas que visem diminuir as dificuldades das crianças com TEA em suas atividades escolares, proporcionando melhor desempenho e desenvolvimento na aprendizagem.

\section{Resultados e Discussão}

Para responder ao problema do estudo, os resultados e discussão foram divididos em tópicos seguindo a mesma sequência do questionário aplicado aos professores, assim, o leitor terá uma melhor interpretação, uma leitura facilitada e dinâmica.

Todos os docentes responderam a primeira pergunta, relacionada a existência de dificuldade dos docentes ao repassar atividade para alunos com TEA (Gráfico 1), no qual (67\%) que corresponde a 2 docentes, de uma amostra de 3 professores, relatam que existir com muita frequência.

Conforme a pesquisa realizada por Bianchi (2017), com uma amostra de 3 docentes, onde as mesmas relatam dificuldade com alunos portadores de necessidade especial, principalmente o TEA, mesmo elas possuindo formação, afirmam que não foi suficiente, para the ajudassem com as dificuldades de sala de aula. Com isso afirmam-se as estatísticas do presente estudo.

Além das pesquisas realizadas e dos dados, presente no estudo também é notável a dificuldade dos docentes na pesquisa realizada por Soares e Mendes(2016), que convém com os dados do presente estudo, pois a mesma foi realizada com uma amostra de 3 docente, no qual os 3 relatam a falta de preparo no trato pedagógico com alunos autistas, além de relatarem que o problema está na falta de formação especifica.

O Gráfico 1 apresenta a existência de dificuldade dos docentes ao repassar atividade para alunos com TEA, no qual (67\%) que corresponde a 2 docentes, de uma amostra de 3 professores, relatam que existir com muita frequência. 


\section{Gráfico 1.}

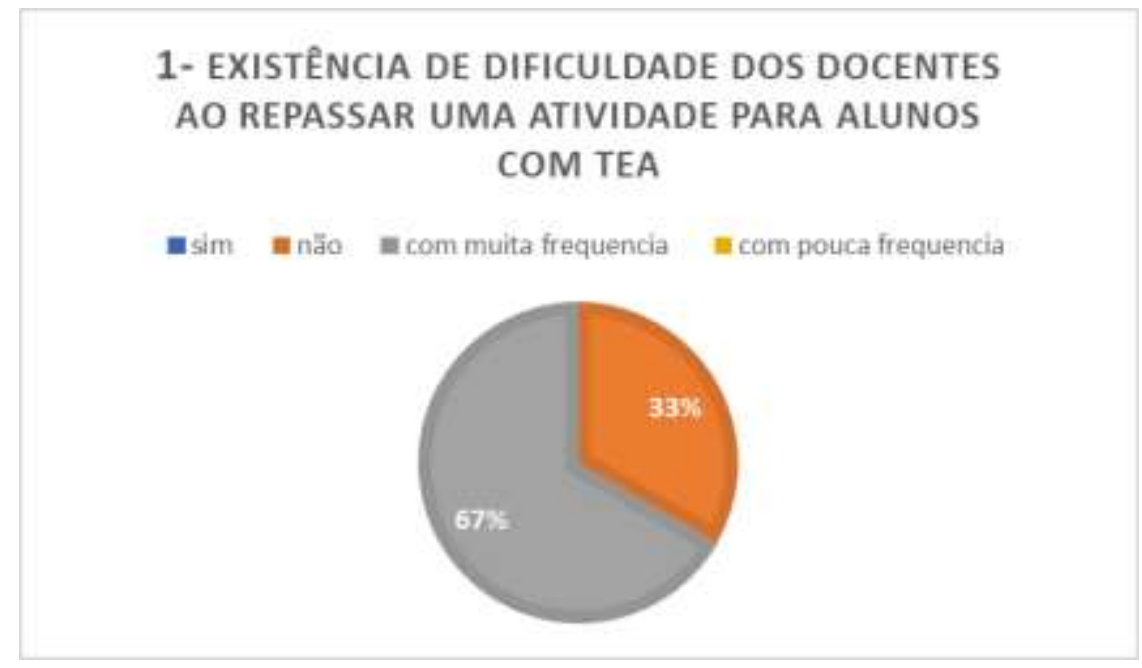

Fonte: Dados da pesquisa (2021).

Nesse tópico demonstra que as crianças com TEA conseguem realizar as atividades sem adaptação adequada, com pouca frequência, segundo $100 \%$ da amostra. Como demonstrado no (Gráfico 2).

Ao realizar algumas pesquisas, pode-se perceber que não só os docentes da escola do presente estudo, como também em outras pesquisas, afirmaram que as crianças com TEA não conseguem ter entendimento de suas atividades sem adaptações adequadas, segundo a pesquisa realizada por Oliveira(2016), em uma amostra de 10 professoras, as mesmas afirmam que seus alunos não recebem adaptações necessárias, sendo assim as professoras (80\%), afirmam que seus alunos com TEA conseguem absorver o conteúdo, apenas 2 docentes (20\%) acreditam que seus alunos conseguem ter apenas uma noção.

Segundo Meireles e Furtado (2019), os alunos com TEA tem capacidade para aprender, faltando assim apenas adaptações que lhe ajudem, pois, eles têm um campo de possibilidades abertas, contando também com o papel dos professores em sala de aula, que estejam preparados e habilitados.

No Gráfico 2, demonstra que as crianças com TEA conseguem realizar as atividades sem adaptação adequada, com pouca frequência, segundo $100 \%$ da amostra.

Gráfico 2.

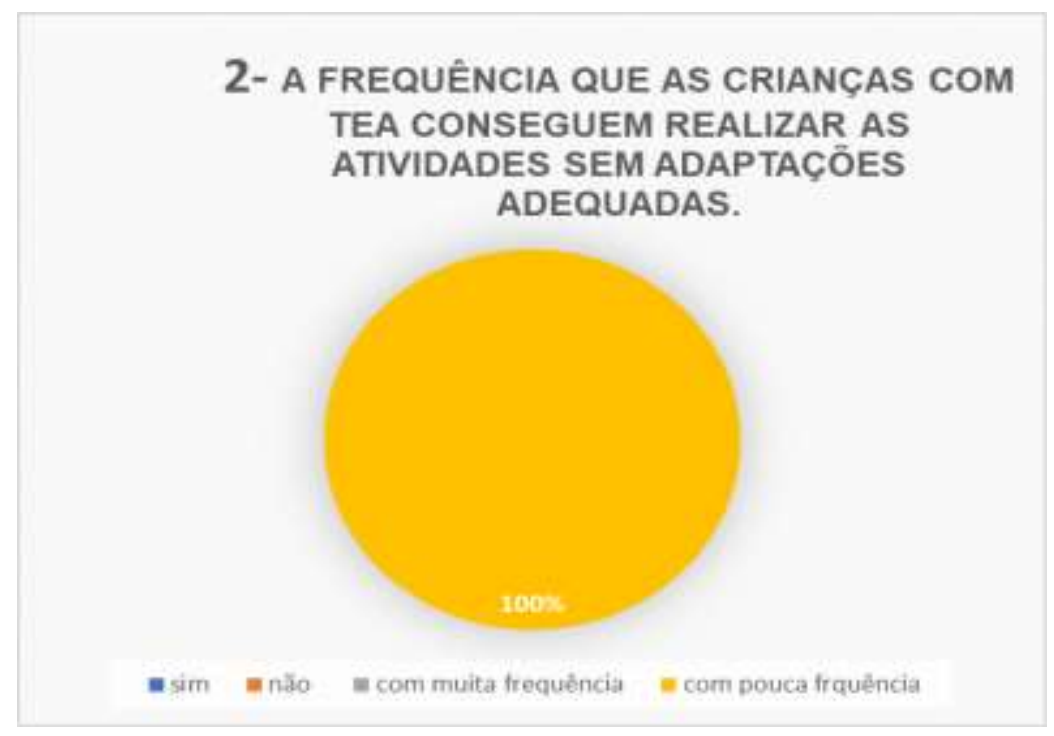

Fonte: Dados da pesquisa (2021). 
Com relação aos docentes possuir alguma formação sobre adaptação curricular, nota-se pouca (67\%) ou nenhuma (33\%) formação para adaptações curriculares para seus alunos com TEA. Como relatado no (Gráfico 3).

Segundo algumas pesquisas feitas, muitos professores possuem dificuldade em sala de aula com alunos com TEA, pois não possuem formação adequada para tais planejamentos de adaptações para seus alunos, pode-se confirmar na pesquisa realizada por (Schmidt et al., 2016) onde afirma-se que existe dificuldade para os professores, onde existem relatos de pouca formação dos docentes (16,2\%), com isso a dificuldade em planejar, ensinar ou avaliar aprendizagens apropriadas a etapa escolar.

A pesquisa de Floro (2019), também é relatado a falta de formação de professores, onde em uma amostra de (10) professoras, seis (6) possuem apenas o curso de Magistério, as outras quatro (4) possuem somente curso superior de Pedagogia, onde relatam a dificuldade com seus alunos, além disso (2) das professoras se quer souberam definir TEA como um transtorno do desenvolvimento, onde ressalta os dados da presente pesquisa.

No Gráfico 3, pode-se perceber que os docentes apresentam pouca (67\%) ou nenhuma (33\%) formação para adaptações curriculares para seus alunos com TEA.

Gráfico 3.

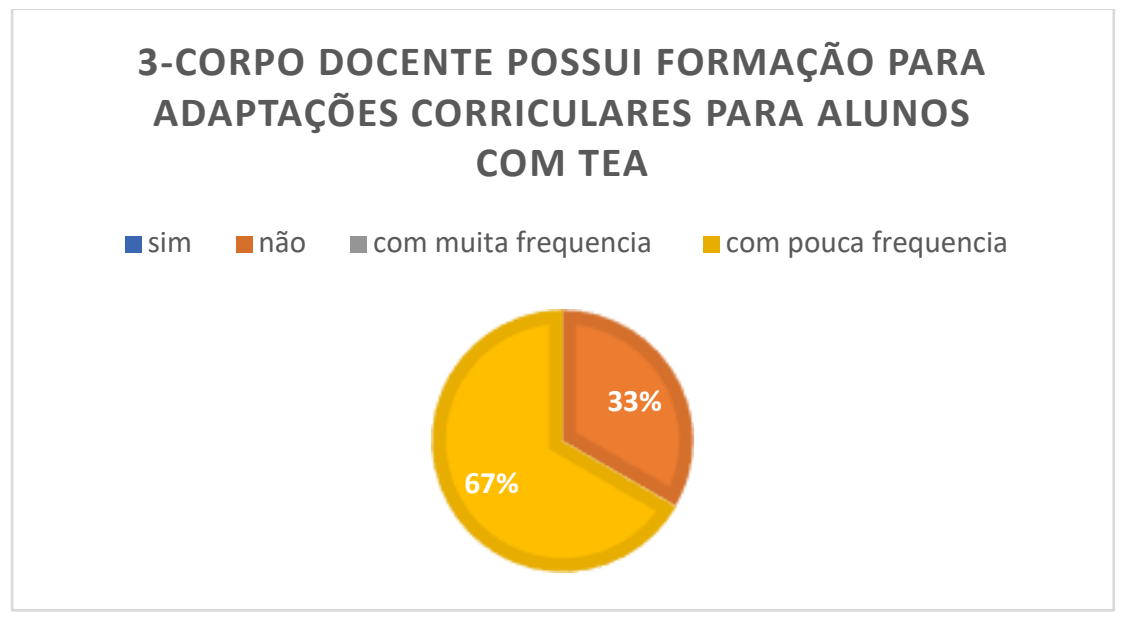

Fonte: Dados da pesquisa (2021).

$\mathrm{Na}$ quarta questão realizada aos professores foi sobre a frequência que a escola disponibiliza materiais que ajudem nas adaptações escolares, foi possível identificar que $67 \%$ da amostra (3 professores), afirmam ser com pouca frequência, dados apresentados no (Gráfico 4).

De acordo com a pesquisa realizada por Oliveira (2016), que levantou dados através de um questionário, pode-se perceber que em nenhuma das 2 escolas de sua amostra foi realizado algum trabalho de adaptação curricular, voltado para crianças com TEA. As entrevistadas (amostra de 10 professoras) alegaram falta de apoio pedagógico das escolas no tocante a realizar algum trabalho diferenciado com crianças diagnosticadas com TEA. O que contrapõe os achados deste estudo, no qual $33 \%$ da amostra relata ter adaptações, e $67 \%$ também relata ter, porém, com pouca frequência.

Observa-se que a pesquisa realizada não é a única que mostra dados sobre a falta de ajuda das escolas em relação a disponibilizar matérias para adaptações das crianças com TEA, segundo a pesquisa realizada por Floro(2019) nenhuma das (2) escolas de sua amostra, realiza algum trabalho de adaptação para seus alunos com TEA, relatando também que os conteúdos de sala de aula são ministrados igualmente para todos, sem nenhuma diferença para alunos com necessidades especiais. 
É possível verificar a frequência com que a escola não disponibiliza de materiais para que ocorra as adaptações para os alunos com TEA, foi possível identificar que $67 \%$ da amostra (3 professores), afirmam ser com pouca frequência, dados apresentados no Gráfico 4.

\section{Gráfico 4.}

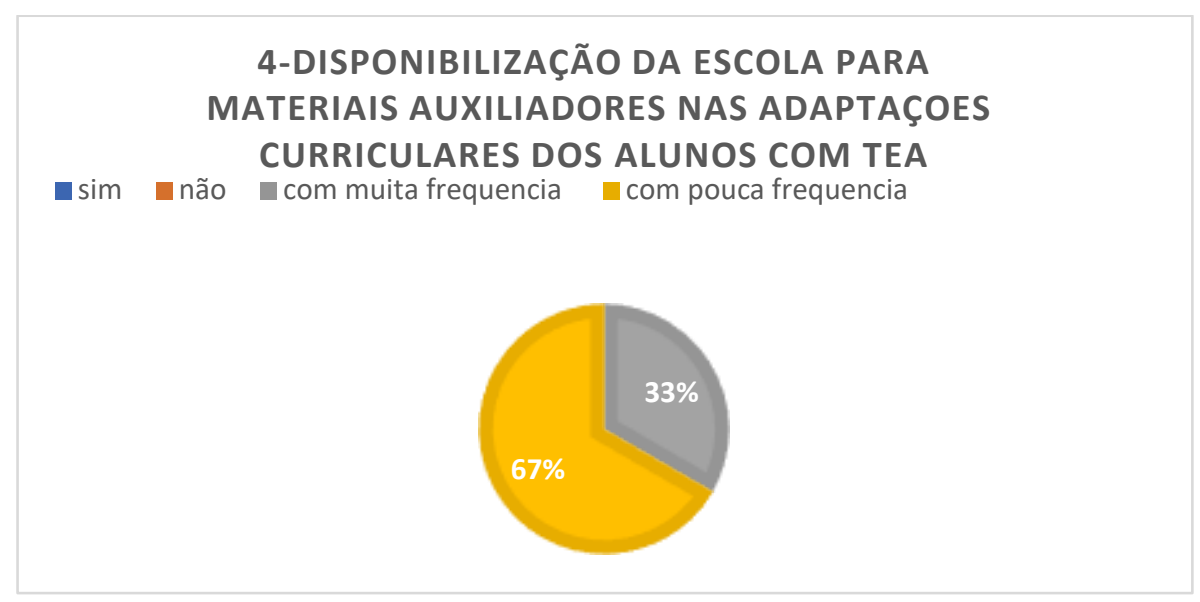

Fonte: Dados da pesquisa (2021).

E por fim foi questionado aos docentes a importância da adaptação curricular para os alunos com TEA. Onde 100\% da amostra deu nota máxima (10). Onde é possível verificar no (Gráfico 5).

Segundo o estudo de caso realizado por Lumertz e Menegotto (2019), a adaptação curricular do aluno mostrou-se uma ferramenta muito importante para a realizar a inclusão escolar do mesmo. O currículo adaptado, além de, individualizado, foi fundamental para que o aluno tivesse acesso ao ensino do qual necessita e tem condições de participar, onde coincide com o nível de importância que é relatado no presente estudo.

Além da importância das adaptações, existe a obrigação da mesma, como a LDBEN assegura em seu artigo 59 inciso I que "os sistemas de ensino assegurarão aos educandos com necessidades especiais: currículos, métodos, técnicas, recursos educativos e organização específica para atender as suas necessidades"

O Gráfico 5, mostra uma escala sobre a importância das adaptações escolares para as crianças com TEA, no qual 100\% da amostra deu nota máxima. 


\section{Gráfico 5.}

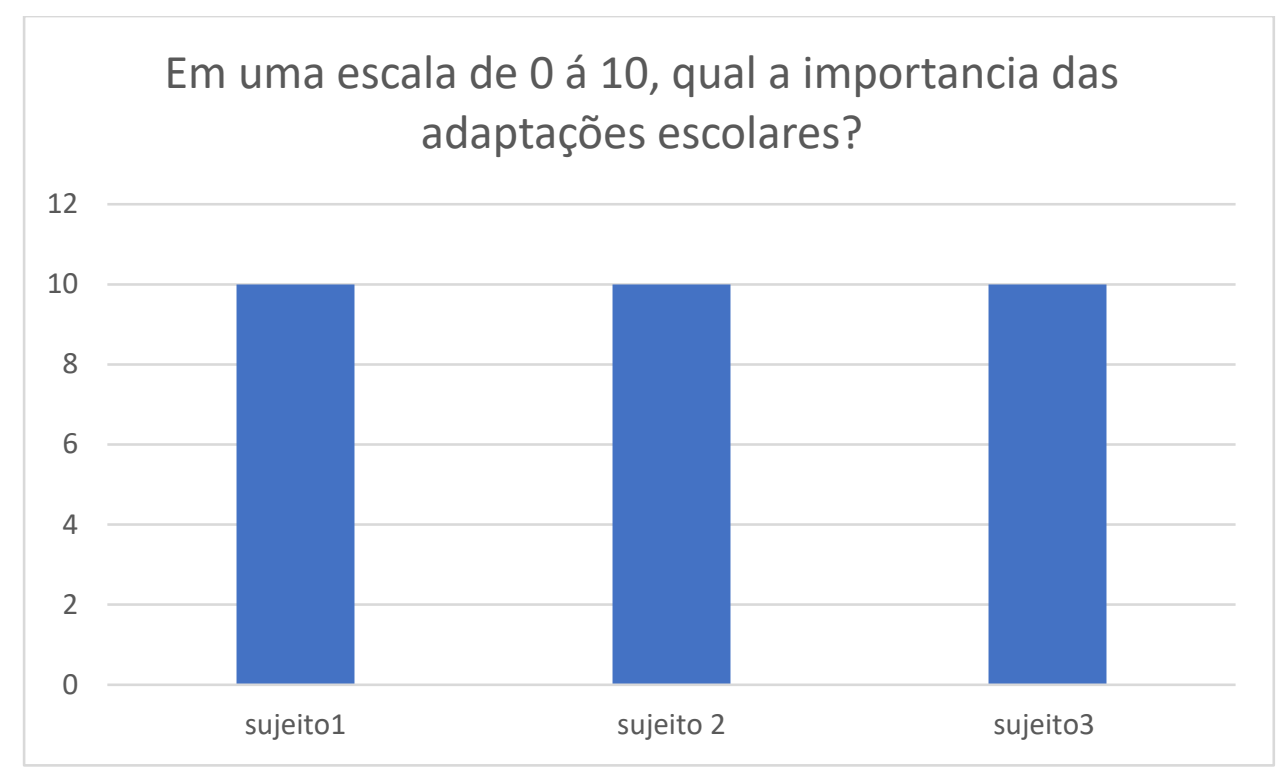

Fonte: Dados da pesquisa (2021).

Segundo o estudo de caso realizado por Lumertz e Menegotto (2019), a adaptação curricular do aluno mostrou-se uma ferramenta muito importante para a realizar a inclusão escolar do mesmo. O currículo adaptado, além de, individualizado, foi fundamental para que o aluno tivesse acesso ao ensino do qual necessita e tem condições de participar, onde coincide com o nível de importância que é relatado no presente estudo.

Além da importância das adaptações, existe a obrigação da mesma, como a LDBEN assegura em seu artigo 59 inciso I que "os sistemas de ensino assegurarão aos educandos com necessidades especiais: currículos, métodos, técnicas, recursos educativos e organização específica para atender as suas necessidades".

\section{Conclusão}

Com base no que foi apresentado sobre às necessidades de adaptação escolar para crianças com TEA, podemos concluir que o presente trabalho é de grande relevância para que possamos analisar as dificuldades encontradas pelos professores no trato com crianças autistas. Apesar de muitas das professoras terem conhecimento do autismo, muitos sentem a necessidade de uma formação mais específica, pois a maioria sente-se despreparada para o trabalho.

Tendo isso em vista, sente-se a necessidade de cursos preparatórios para os docentes para que assim o professor aprenda a lidar com as diferenças de sua sala de aula, afastando os temores, a ansiedade, as ideias pré-concebidas, fazendo com que haja inclusão de crianças autistas nos anos iniciais do Ensino Fundamental, já que muitas crianças com TEA têm um ritmo de aprendizagem diferente, logo, há necessidade de instrumentos de avaliação diferenciados.

Assim, vale ressaltar a importância de uma maior divulgação sobre esse tema, e a necessidade de novos estudos para ampliar o conhecimento em torno do assunto, considerando que é indispensável conhecimento em escolas, para os familiares e para a sociedade para que viabilizem o acolhimento e a compressão em relação à condição do autista.

\section{Referências}

Amato, C. A.H., \& Fernandes. F. D. M. (2010). O uso interativo da comunicação em crianças autistas verbais e não verbais. Pró-Fono R. Atual. Cient., 22(4), 373-378

Benini, W, \& Castanha, A. O. (2016). A inclusão do aluno com transtorno do espectro autista na escola comum: desafios e possibilidades. Cadernos PDF. 
Bianchi, R. C. (2017). A Educação De Alunos Com Transtornos Do Espectro Autista No Ensino Regular: Desafios E PossibilidadeS. Universidade estadual paulista. Franca/SP.

Camargo, S. P. H., Silva, G. L., Crespo, R. T., Oliveira, C. R., \& Magalhães, S. L. (2020). Desafios No Processo De Escolarização De Crianças Com Autismo No Contexto Inclusivo: Diretrizes Para Formação Continuada Na Perspectiva Dos Professores. Educ. rev. vol.36

Corrêa, P. M, et al. (2017). A importância da Terapia Ocupacional no brincar da criança com autismo. Ling. Acadêmica, 7(7), 37-55.

2019 CREFITO-9- Conselho Regional De Fisioterapia E Terapia Ocupacional Da $9^{\circ}$ Região.

Cunha, E. (2017). Autismo e inclusão: psicopedagogia práticas educativas na escola e na família. Ed. 2017.

Ferreira, V. S, et al. (2020) O transtorno do espectro autista e a inclusão escolar: contribuições para o desenvolvimento da criança.

Floro, A. P. O. (2019) Adaptação curricular para autistas no Ensino Fundamental 1: Um enfoque na Legislação Educacional. Revista Sociedade em Debate. Conselho de ensino e Extensão- Faculdade Três Marias. 1(1).

Gomes, R. F, \& Onzi, F. Z. (2015) Transtorno Do Espectro Autista: A Importância Do Diagnostico E Reabilitação. Caderno Pedagógico, 12(3), 188-199.

Marteleto, M. R.F, et al. (2011) Problemas de Comportamento em Crianças com Transtorno Autista. Universidade Federal de São Paulo. Departamento de Distúrbios da comunicação Humana. Rua Botucatu, 802- Vila Clementino. Psicologia, Teoria e Pesquisa. 27(1), 5-12.

Meirelles, et al. (2019) Educação Inclusiva: Adaptações Curriculares Para O Aluno Autista. Revista Interdisciplinar do Pensamento Científico. 5(5).

Melo, M. M, et al. (2019) Atendimento multidisciplinar para a educação especial e inclusiva de uma criança com transtorno do espectro autista: um estudo de caso. Revista Eletrônica Acervo Saúde/ Eletrônica Jornal Collection Health.REAS/EJCH, 25.

Minatel, M. M., \& Matsukura, T. S. (2014). Famílias de crianças e adolescentes com autismo: cotidiano e realidade de cuidados em diferentes etapas do desenvolvimento. Rev. Ter Ocup Univ. São Paulo. 25(2), 126-34.

Neves A. J, et al. (2014). Escolarização Formal E Dimensões Curriculares Para Alunos Com Autismo: O Estado Da Arte Da Produção Acadêmica Brasileira. Educação em Revista Belo Horizonte. 30(2). 43-70.

Oliveira. A. N. (2016). Adaptação curricular para autistas no ensino fundamental I: um enfoque na legislação educacional. Centro de educação da Universidade Federal da Paraiba.

Onzi, F. Z., \& Gomes, R. F. (2015). Transtorno do espectro autista: a importância do diagnóstico e reabilitação. Caderno pedagógico, 12(3), 188-199.

Pinto, R. N. M, et al (2016) Autismo infantil: impacto do diagnóstico e repercussões nas relações familiares. Rev Gaúcha Enferm. (3): e61572.Versão online Português/Inglês:www.scielo.br/rgenf.

Rosa, V. I, Silva, R. P., \& Aymone, J. L. F. (2018) Design inclusivo: processo de desenvolvimento de prancha de comunicação alternativa e aumentativa para crianças com transtorno do espectro do autismo utilizando realidade aumentada. Design e Tecnologia, 8(15), 51-67.

Schmidt, Carlos. (2017) Transtorno Do Espectro Autista: Onde Estamos E Para Onde Vamos. Psicologia em Estudo, 22(2), 221-230.

Schmidt, Carlos, et al. (2016) Inclusão escolar e autismo: uma análise da percepção e práticas pedagógicas. Revista Psicologia: Teoria e Pratica, São Paulo.

Serra.D. (2010) Autismo, Família E Inclusão. Polêm!ca, 9(1), 40 - 56.

Silva, W. N, \& Freitas, F. P. M. (2016) Atividades de Adaptação Curricular para Crianças Com transtorno do espectro Autista na perspectiva do programa teacch: relato de experiência. Revista Diálogos e Perspectivas em Educação Especial, 3(2), 117-126.

Soares. M. P., \& Mendes. G. G. (2016) As principais dificuldades dos professores de educação física na inclusão de alunos com autismo: Uma Análise da rede Municipal de Educação de Criciúma/ SC.

Souza, A. J. et al. (2019) Anais Da Iv Semana De Letras Da Urca: Linguagem E Ideologia: Reprsentações Possiveis Entre Lingua E Literatura. Missão Velha: Edoc Brasil, 2019.

Lumertz. F. D. S, \& Menegotto. L. M. O. (2019) Adaptação Curricular Como Instrumento De Inclusão Escolar De Um Aluno Com Tea: Relato De Experiência. Revista Gepesvida, 13(5).

Velloso. R. L, et al. (2011) Protocolo De Avaliação Diagnóstica Multidisciplinar Da Equipe De Transtornos Globais Do Desenvolvimento Vinculado À PósGraduação Em Distúrbios Do Desenvolvimento Da Universidade Presbiteriana Mackenzie. Universidade Presbiteriana Mackenzie. Cadernos de Pós-Graduação em Distúrbios do Desenvolvimento, 11(1), 9-22.

Zanon, R. B, Backes, B, \& Bosa. C. A. (2014) Identificação Dos Primeiros Sintomas Do Autismo Pelos Pais. Psicolofia: Teoria e pesquisa. 30(1), 25 